\title{
1 Assessing and removing the effect of unwanted technical 2 variations in microbiome data
}

3 Muhamad Fachrul ${ }^{1,2}$, Guillaume Méric ${ }^{1,3}$, Michael Inouye ${ }^{1,2,4,5,6,7,8}$, Sünje Johanna Pamp ${ }^{9,10}$, 4 Agus Salim ${ }^{11,12,13,14^{*}}$

$5 \quad{ }^{1}$ Cambridge Baker Systems Genomics Initiative, Baker Heart and Diabetes Institute, Melbourne, VIC 63004 , Australia.

$7 \quad{ }^{2}$ Department of Clinical Pathology, University of Melbourne, Parkville, VIC 3010, Australia.

$8{ }^{3}$ Department of Infectious Diseases, Central Clinical School, Monash University, Melbourne, VIC 3004, 9 Australia.

$10{ }^{4}$ Cambridge Baker Systems Genomics Initiative, Department of Public Health and Primary Care,

11 University of Cambridge, Cambridge, UK

$12{ }^{5}$ British Heart Foundation Cardiovascular Epidemiology Unit, Department of Public Health and Primary

13 Care, University of Cambridge, Cambridge, UK

$14{ }^{6}$ British Heart Foundation Centre of Research Excellence, University of Cambridge, Cambridge, UK

$15{ }^{7}$ Health Data Research UK Cambridge, Wellcome Genome Campus and University of Cambridge,

16 Cambridge, UK

$17 \quad{ }^{8}$ The Alan Turing Institute, London, UK

$18{ }^{9}$ National Food Institute, Technical University of Denmark, 2800 Kgs. Lyngby, Denmark.

$19{ }^{10}$ Novo Nordisk Foundation Center for Biosustainability, Technical University of Denmark, 2800 Kgs.

20 Lyngby, Denmark.

$21{ }^{11}$ Centre for Epidemiology and Biostatistics, Melbourne School of Population and Global Health, The

22 University of Melbourne, Melbourne, VIC 3010, Australia.

$23{ }^{12}$ School of Mathematics and Statistics, The University of Melbourne, Melbourne, VIC 3010, Australia.

$24{ }^{13}$ Department of Population Health, Baker Heart and Diabetes Institute, Melbourne, VIC 3004, Australia.

$25{ }^{14}$ Department Mathematics and Statistics, La Trobe University, Bundoora, VIC 3086, Australia.

27 *Correspondence: salim.a@unimelb.edu.au

28 Keywords: unwanted variations, microbiome, bioinformatics, batch effects. 


\section{Abstract}

30 Varying technologies and experimental approaches used in microbiome studies often lead to

31 irreproducible results due to unwanted technical variations. Such variations, often unaccounted

32 for and of unknown source, may interfere with true biological signals, resulting in misleading

33 biological conclusions. In this work, we aim to characterize the major sources of technical

34 variations in microbiome data and demonstrate how a state-of-the art approach can minimize their

35 impact on downstream analyses. We analyzed 184 pig faecal metagenomes encompassing 21

36 specific combinations of deliberately introduced factors of technical and biological variations. We

37 identify several known experimental factors, specifically storage conditions and freeze-thaw

38 cycles, as a likely major source of unwanted variation in metagenomes. We also observed that

39 these unwanted technical variations do not affect taxa uniformly, with freezing samples affecting

40 taxa of class Bacteroidia the most, for example. Additionally, we benchmarked the performance

41 of a novel batch correcting tool used in this study, RUV-III-NB

42 (https://github.com/limfuxing/ruvlllnb/), to other popular batch correction methods, including

43 ComBat, ComBat-seq, RUVg, and RUVs. While RUV-III-NB performed consistently robustly

44 across our sensitivity and specificity metrics, most other methods did not remove unwanted

45 variations optimally, with RUVg even overcorrecting and removing some of the true biological

46 signals from the samples. Our analyses suggests that a careful consideration of possible technical

47 confounders is critical in the experimental design of microbiome studies to ensure accurate

48 biological reading of microbial taxa of interest, and that the inclusion of technical replicates is 49 necessary to efficiently remove unwanted variations computationally. 


\section{Introduction}

52 The technological advancement of sequencing technologies has made microbiome studies more

53 accessible and meaningful. From amplifying short 16S rRNA hypervariable regions to taking

54 advantage of long-read sequencing, the breadth of data options has enabled the field to flourish

55 in the past couple of decades, allowing a better understanding of the role of microbiomes in

56 numerous ecological, environmental and clinical contexts ${ }^{1}$. For example, the dynamics of the gut

57 microbiome is now known to be influenced by environment and diet, and perturbations (or

58 "dysbiosis") have been linked to chronic conditions such as cardiometabolic diseases and type 2

59 diabetes $(\mathrm{T} 2 \mathrm{D})^{2-4}$. As a result, the human microbiome is now considered an important biological

60 basis for potential therapeutic targets ${ }^{5,6}$. However, the multiplication of microbiome studies using

61 a very wide range of technological methods and experimental designs comes with a cost on

62 reproducibility. Any valuable application from microbiome studies has indeed been largely

63 hindered by the lack of reproducibility due to the presence of unwanted technical variations.

64 Microbiome studies can differ considerably when it comes to the experimental approaches; and 65 each step of the workflow, including variations introduced by the experimenter, has the potential 66 to introduce artificial results due to unwanted technical variations ${ }^{7,8}$. For example, under-sampling 67 might occur due to the lack of consideration during initial collection process, resulting in zero 68 reads detected for certain microbiota that actually exist in the environment due to their 69 underrepresentation in the samples ${ }^{9}$. Additionally, specific stool collection kits have been shown 70 to impact microbial abundance of faecal samples differently ${ }^{10}$. DNA extraction, library preparation

71 kits, storage condition, storage time, and choice of sequencing platforms have also been found 72 to introduce artificial variations in microbial abundance ${ }^{7,8,11,12}$. As an example, outgrowth of certain 73 microorganisms has been reported between sample preservation methods, as some methods are 74 unable to prevent the growth of facultative anaerobic organisms ${ }^{12}$. Contamination of external 75 microbial taxa can also contribute to unwanted variations and can happen at any stage of the 76 experiment. For instance, recent studies have found that some library preparation kits used before 77 sequencing could introduce specific microbial taxa coming from reagents in the $\mathrm{kit}^{13}$. This issue 78 is observed to be even more critical in low-biomass samples, even leading to debated 79 interpretations on the existence of a microbiome in environments that might not have any, such 80 as the human placenta or the meconium ${ }^{13-16}$. Left unaddressed, such unwanted variations can 81 considerably confound true biological signals and result in misleading conclusions.

82 Various computational methods have been developed to correct for batch effects within 83 experiments. Popular methods such as ComBat ${ }^{17}$ and RUV ${ }^{18}$, originally developed for microarray 
84 and RNA-seq transcriptomics datasets, have also been considered for microbiome studies ${ }^{7,19}$ but $^{1}$

85 do not particularly take into account the specific characteristics of microbiome data such as

86 compositionality and zero-inflation. Indeed, contrary to features in transcriptomics datasets, the

87 presence of each taxa is not independent from the rest of the taxa in the microbiome, and raw

88 abundance information acquired after sequencing is not directly representative of the actual

89 abundance in the environment due to the limit of the sequencing depth that each platform has ${ }^{20}$,

90 a problem that is not commonly addressed in current studies ${ }^{21}$. Additionally, microbiome data are

91 typically zero-inflated and very sparse, as many features are present in only a very few samples.

92 Log-transformation of raw abundances, a process allowing for more robust statistical analyses to

93 be performed, prerequires to substitute zero-values with a constant arbitrary number, also known

94 as "pseudocount". Despite being part of the compositional data analysis (CoDA) standards, there

95 is an argument against adding pseudocount then using log-transformation in analyzing highly

96 sparse datasets in the form of counts per sample, as it changes the ratio of taxa abundance

97 substantially, diminishes variance from less abundant taxonomic groups and artificially

98 exaggerates the differences between zero and non-zero values ${ }^{22,23}$.

99 In this study, we aim to characterize the major sources of technical variations in microbiome data

100 and demonstrate how a state-of-the art approach can minimize their impact on downstream

101 analyses. Using a dataset containing 184 faecal microbiome samples from pigs comprising up to

10221 unique combinations of technical variations, we use RUV-III-NB

103 (https://github.com/limfuxing/ruvllInb/), a novel robust batch correction tool which utilizes

104 Negative Binomial (NB) distribution to estimate and adjust for unwanted variations without the

105 need to add pseudocounts, to identify parts of the experimental workflow that introduce critical

106 unwanted variations that affect observed microbial abundances. Then, we compare the

107 performance of RUV-III-NB to other popular tools including ComBat, ComBat-Seq, RUVg, and

108 RUVs. 


\section{Results}

110 For the main analyses of assessing the contributions of unwanted variations towards microbial

111 abundance, we utilized the novel RUV-III-NB, which is able to estimate and adjust sequencing 112 count data for unwanted technical variations based on the negative control taxa and sample 113 replicate information in the dataset. For benchmarking purposes, we refer to methods utilizing 114 batch information for control measure (ComBat and ComBat-Seq) as ComBat-based methods, 115 and methods utilizing control features (RUVg, RUVs, and RUV-III-NB) as RUV-based methods.

\section{Unwanted variations correlate with known sources of technical variations, and} 117 mostly affect highly abundant taxa.

118 We acquired 7 unwanted factors (referred to as W1 to W7) from using RUV-III-NB on the spiked 119 pig metagenomes, representing detected sources of unwanted variations within the dataset. The 120 primary unwanted source of variation (called W1) correlated with log library size ( $r=0.648$ ), which 121 suggests that it is capturing the variability caused by sequencing depths between samples. It also 122 correlated with log geometric mean ( $r=0.964)$, highlighting the ability of RUV-III-NB to identify the 123 optimal form of normalization. In this case, it supports the method of utilizing log geometric mean 124 as scaling factor, such as with the centered log-ratio (CLR) transformation, as a more effective 125 approach than total sum scaling (TSS) normalization at providing the most accurate abundance 126 estimate. A number of known experimental factors were also found to correlate well with other 127 unwanted variations: we found storage conditions and freeze-thaw cycles correlating with W2 $128(r=0.770)$ and W3 ( $r=0.782)$, respectively (Figure 1B). Differences in the library preparation kit 129 used were also found to weakly correlate with W5 ( $r=-0.522)$ and W6 ( $r=0.500)$.

130 Microbial taxa belonging to the Bacteroidia class were found to be the most affected by W2, which 131 correlated to storage conditions of the samples (Figure 1C). Members of the Bacilli and Clostridia 132 classes were also among the most affected across W3-W7. Incidentally, Bacteroidia, Bacilli, and 133 Clostridia were the most abundant classes in the dataset, with 2,153 species belonging to 134 Bacteroidia, totaling over 656 million reads (62.8\% of total). To estimate the extent to which each 135 of the detected unwanted factor affected our samples, we calculated the proportion of read counts 136 belonging to the 100 most affected taxa, defined by the taxa with the highest pseudo- $\mathrm{R}^{2}$ for each 137 unwanted factor (Figure 1D). We found that storage conditions (referring here whether samples were kept frozen or at room temperature before processing), represented by unwanted factor W2, affected up to $25.32 \%$ of a sample's total abundance - a notable amount considering only 100 out of 8,453 taxa were taken into account (Figure 1D). This suggests that after library size, storage conditions are the primary source of unwanted variation in microbiome studies. Unwanted 
142 factors W3 and W4 contributed to $4.72 \%$ and $4.29 \%$ of overall sample abundance, respectively,

143 whereas W5-W7 each contributed to under $2 \%$.

\section{Technical variations do not affect different taxa uniformly.}

145 The previous observation suggests that not every taxon is affected similarly by variations in 146 technical procedures, and that some taxa are biologically more sensitive or resistant to varying experimental conditions than others. To further this, we analyzed abundances from several microbial classes in samples from the same source (P1) but subjected to different technical variations (Figure 1A). We found the highly abundant bacterial classes to still be differentially abundant between batch groups, including storage conditions, freeze-thaw cycles, and library preparation kits. Taxa from classes Bacteroidia, Bacilli, Clostridia, and Alphaproteobacteria were found to significantly differ in abundance when compared between storage conditions and freezethaw cycles, with taxa from Alphaproteobacteria also differed between samples generated from different library preparation kits (Figure S1). Moreover, the differential abundance patterns, as in the state of the taxa in question being more abundant in one condition than the other, were not shared between bacterial classes in the same conditions: Bacteroidia was found to be more abundant in unfrozen samples, whereas Bacilli, Clostridia, and Alphaproteobacteria were less abundant. The pattern also differed for these classes in samples generated with varying freezethawing cycles and library preparation kits, with Bacilli, Clostridia sharing a similar abundance pattern across the different cycles. This suggests that the effect of technical variations is not uniform across different taxa.

\section{Most RUV-based methods remove unwanted variations better than ComBat-based} methods

164 We then plotted relative log expression (RLE) plots before and after correction using ComBat, ComBat-seq, RUVg, RUVs, and RUV-III-NB, to assess how much overall unwanted variations were removed from each approach, by calculating a metric based on the variance of the medians and interquartile ranges. Unlike with factors of unwanted variations or principal components (PCs) where we could assess how each of them correlates with specific technical variations, RLE plots only assess the whole/overall unwanted variations that exist within a dataset; hence, the term overall unwanted variations here is used when referring to results from RLE analysis. The RLE

171 plots and principal component analysis (PCA) plots before and after RUV-III-NB correction

172 (Figure 2A, Figure 2B) visually represent the removal of overall unwanted variations. Based on 173 the RLE metric, better corrections were seen for RUV-based methods than ComBat-based 174 methods, with the exception of RUVs using spike-in taxa only (Figure 2C). RUVg had the best 
175 RLE metric in average ( $\bar{x}$ rle=8.64), followed by RUV-III-NB ( $\bar{x}$ rle=6.95). RUVs removed unwanted variations comparably to RUV-III-NB when using both combination and only empirical sets of control features yet did not remove much overall unwanted variations when using only spike-in taxa (rle=0.49), scoring even lower than uncorrected data (log2 rle=2.93, CLR rle=3.98). Both tested ComBat methods, using log2 and CLR transformations, had RLE metrics higher than uncorrected data, but still scored lower on average $(\bar{x}$ rle=4.58) than the lowest-scoring $R U V g$ $(r l e=5.15)$ and RUV-III-NB (rle=4.98) runs. ComBat-Seq did not seem to remove the entirety of the unwanted variations as the resulting RLE metric was similar to uncorrected data with log2 transformation $(r l e=2.94)$. This analysis suggests that RUV-based methods generally perform better in removing overall unwanted variations in microbiome data than ComBat-based methods.

To get a visual representation of overall variation in datasets and the effects of correcting for batch effects, we performed principal component analysis (PCA) on the microbiome composition matrix data and calculated the silhouette statistics (ss) for clustering by a technical factor of main batch separation (in this case storage conditions), using the top four principal components (PCs) before and after correction (Figure 2B). Specific clustering of samples based on storage conditions and the library preparation kit used could be seen in the PCA plots before any batch effect correction, specifically captured by PC3 and PC4, respectively, but this was no longer apparent after correction with RUV-III-NB using both spike-in and empirical taxa (Figure 2B). With the exception of RUVs using only empirical control taxa $(s s=0.51)$, all approaches had lower silhouette scores compared to uncorrected data (uncorrected $s s=0.488$; ComBat $s s=0.11-0.26$; ComBat-Seq $s s=0.11 ; R U V g s s=0.14-0.17$; RUV-III-NB $s s=0.09-0.16$; RUVs $s s=0.08-0.51$ ), suggesting successful removal of storage condition effects (Figure S2). The effect of using different normalization methods did not seem to alter the silhouette scores in uncorrected data, unlike when using ComBat approaches. Overall, correction using RUV-III-NB and ComBat-Seq yielded the lowest average scores within this dataset, indicating their robustness in removing unwanted variations from the major technical variation source.

201 To identify taxa most affected by different unwanted sources of variation, we performed differential 202 abundance analysis using edgeR between the different storage conditions, in which only spiked 203 P1 samples were used. After FDR correction and fold change filtering (FDR< $0.05,|\log 2(F C)|>1)$, 204 samples ran using ComBat-Seq had the lowest number of differentially abundant taxa after 205 correction, followed by RUV-III-NB and RUVs with combination control taxa (Table S1). Yet, 206 correction with RUV-III-NB resulted in the highest estimates of the proportion of null (pi0; non207 differentially expressed) features, with correction using combination and solely empirical taxa 
reaching 0.964 and 0.950, respectively - higher than ComBat-Seq (pi0=0.905) (Figure 2D). Correction using RUV-III-NB also resulted in the highest average pi0 compared to the other RUV methods, followed by RUVs which performed comparably when using combination control features (pi0=0.955). RUV methods with solely spike-in control taxa all performed comparably,

212 with higher results than uncorrected data but lower than ComBat-Seq. Taken together, these

213 observations suggest that RUV-III-NB performed the most consistently robust compared to the

214 other methods in removing unwanted variations.

\section{Technical replicates and choice of control features are crucial for RUV methods}

216 To assess how well correction methods preserve individual information, we calculated silhouette 217 statistics of individual separation after removal of unwanted variations, a metric describing how 218 well samples from two conditions (here, pigs P1 and P2) separate after clustering, based on how 219 close each point in one cluster is to points in the neighboring clusters; in this case, high value 220 indicates precise clustering and separation of samples based on the pig individuals and, whereas 221 low value suggests otherwise. RUVs using empirical control taxa had the highest silhouette score 222 (ss=0.797), followed by RUVs and RUV-III-NB with combination control taxa (ss=0.790 and 0.761 , 223 respectively) (Figure 3A). However, RUV-III-NB in average had a higher silhouette score than 224 RUVs, as correction with solely spike-in control taxa using the latter yielded a silhouette score 225 lower than uncorrected data (RUVs $\bar{x} s s=0.64$, RUV-III-NB $\bar{x} s s=0.75$ ). ComBat-based methods 226 performed comparably to uncorrected data, with correction using ComBat using log2 having 227 higher silhouette score. Interestingly, RUVg yielded the lowest score overall, with all three control 228 feature options scoring just below zero. This is visualized in the PCA plots before and after 229 correction, in which separation of $\mathrm{P} 1$ and $\mathrm{P} 2$ samples are explained by PC1 and PC2 even before 230 any correction. The discrimination between individual metagenomic profiles is retained after RUV231 III-NB correction, but not after using RUVg (Figure 3B). This analysis suggests that sample 232 replicate information is necessary when correcting unwanted variations using RUV-based 233 methods to avoid over-correction. That being said, despite both methods utilizing sample replicate 234 information, RUV-III-NB still prevailed over RUVs when taking into consideration performance 235 consistency over the different sensitivity and specificity metrics.

\section{Discussion}

237 The impact of technical variations in microbiome analysis is an important topic that has been 238 explored numerous times, and past studies have reported on the best practices at every stage of 239 sample processing ${ }^{24}$. However, our study is the first to quantify the contribution of different 240 technical factors towards unwanted variations in a shotgun metagenomic sequencing dataset, 
241 and how to best remove them. To do so, we used RUV-III-NB, a novel tool accessible on

242 (https://github.com/limfuxing/ruvIIInb/), which we also compare to other available published

243 methods.

244 Inconsistent sample storage can be a large source of unwanted variations in microbiome studies

246 The variety of storage conditions - which encompass factors such as temperature, time, and 247 storing method used - have been previously found to introduce considerable effects on microbial 248 abundance ${ }^{11,12,25,26}$. The impact of different storage conditions is also not limited to metagenomic 249 data, as Hickl et al. also reported significant differences in microbial protein identification between 250 samples stored using flash freezing and RNAlater ${ }^{27}$. Our study does not aim to replicate and 251 validate the findings of such studies, but to show how, if left unaddressed, the differences in 252 sample storage conditions could introduce significant changes in microbial abundance of 253 abundant taxa which are potentially important for our understanding of particular conditions or 254 diseases. The three most-affected bacterial classes in our study, namely Bacteroidia, Clostridia 255 and Bacilli, are among the most dominant microbial classes in the mammalian gut ${ }^{28-30}$, therefore 256 it is not surprising to see them strongly affected by technical variations. Both Bacteroidia and 257 Clostridia classes in particular have been found to be significantly impacted by different storage 258 methods in both metagenomic and metaproteomic data ${ }^{27}$. In clinical cases, the dysbiosis of 259 members of the classes Bacteroidia and Clostridia have been linked to a wide variety of 260 conditions, including T2D, Crohn's disease, lupus, HIV infection, and major depressive 261 disorders ${ }^{30-35}$. Hence, extra precautions are necessary in making sure the experimental design 262 minimizes sources of unwanted variations as possible.

\section{Metagenomic sequencing depth and data normalization also contribute to}

\section{4 unwanted variations in microbiome studies}

265 Aside from storage conditions, RUV-III-NB identified that library size and geometric mean correlated with unwanted factor $\mathrm{W} 1$ in our study, which is unsurprising. In microbial

267 metagenomics, sequencing depth typically impacts the robust classification of reads into genes 268 or microbial taxa, and optimal depth to use depends on each study objectives ${ }^{36,37}$. Our dataset 269 included a range of sequencing depths, which explains why library size was captured as a source 270 of variation by RUV-III-NB. Furthermore, although normalization and log transformation alone are 271 not enough to remove unwanted variations, normalization by library size, also known as TSS 272 normalization, remains widely used in the field mostly due to conceptual simplicity ${ }^{38}$. However, 
273 this approach does not account for heteroskedasticity and is very sensitive to pseudocount ${ }^{39}$. With

274 unwanted factor W1 correlating much stronger with geometric mean than library size, our work

275 suggests that normalizing by geometric mean (e.g., CLR transformation) rather than by library

276 size (e.g. TSS) potentially limits unwanted variations in observed microbial abundances.

\section{RUV-III-NB is a robust and consistent microbiome batch correction method}

278 Although the observation of best practice methods and preventive measures are always recommended when it comes to avoiding unwanted technical variations in any experimental setting, there are a few existing computational approaches to rectify batch effect variation after a dataset has been processed and sequenced. Here, we show that, despite deliberately introduced complications in our gut metagenomics experimental workflow, the effective removal of unwanted variations was still possible during downstream analysis, with different methods varying in efficiency. Methods such as ComBat, RUVg, and RUVs require normalization of the data prior to the actual batch removal step, but RUV-III-NB is able to take into account the most optimal normalization method and identify unwanted variations in one step without any prior pseudocount addition.

Additionally, ComBat-based methods rely on known batch information and might not be suitable in the realistic situation of multiple batch effects conflating with true biological signals. Nevertheless, when batch information is clearly defined, ComBat-based methods remove unwanted variations efficiently from our samples, but also impact the recovery of true biological signals separating individuals. In our work, ComBat-based methods were also unable to identify and adjust for unknown unwanted variations, which are expected to be common and unavoidable in reality. RUV methods avoid this hurdle by adjusting samples based on the variations of the control features, which are assumed to be in constant amount across technical variations.

One potential issue with RUV methods is that, unlike with RNA-seq data where housekeeping genes have been established ${ }^{40,41}$ and are routinely used to normalize expression, there is no obvious parallel in microbiome features, as taxa composition varies considerably across datasets.

299 The choice of control features is especially crucial for the methods to work as intended; therefore, 300 the presence of spike-ins is highly beneficial when utilizing RUV methods in microbiome studies. 301 In our case, the mock community consisted of 6 bacterial and 2 eukaryotic microorganisms. Over 3023,000 genes have been established to be consistently expressed in human cells and are used to 303 normalize expression in RNA-seq studies, which is considerably larger than our spike-ins. We 304 therefore supplemented the spike-in taxa with a set of empirical control taxa. In this study, 305 correction with only the spike-in control taxa removed unwanted variations to an extent, though 
using the additional empirical control taxa significantly improved it. Additionally, we observed that

307 using only empirical taxa without spike-ins was still effective in removing unwanted variations,

308 indicating that it is possible to effectively use RUV methods without spike-in taxa, which is more

309 convenient when preparing samples.

310 When comparing between RUV methods, we showed how inclusion of sample replicate

311 information is important, as demonstrated by RUVg's tendency to overcorrect and lose individual

312 separation in the process. Both RUVs and RUV-III-NB utilize sample replicate information, and

313 are therefore able to avoid overcorrection, yet the former performed less consistent across the

314 performance tests. RUV-III-NB has an advantage over RUVs due to its direct modelling of mean-

315 variance relationship of count data - a feature not shared by RUVs.

316 In this study, we show how technical variations - especially storage conditions - may introduce

317 unwanted variations in microbiome data, affecting the observed abundances of important and

318 dominant microbial taxa. Left unaddressed, this might be problematic for studies focusing on such

319 taxa, therefore careful considerations are crucial in designing the experimental workflow of

320 microbiome studies. Minimizing the possibility of introducing unwanted variations by limiting the

321 presence of batches and utilizing consistent storage conditions and equipment are highly

322 suggested. We finally show that for existing datasets, post-processing corrective measures can

323 still be performed in silico to remove unwanted variations stemming from variations in

324 experimental techniques, and we suggest the use of RUV-III-NB as a consistent and robust 325 method. 


\section{Materials and Methods}

\section{Sample processing, library preparation, and sequencing conditions}

329 The fecal samples investigated in this study originated from two individual pigs (P1 and P2) 330 collected right after defecation from two different conventional pig farms in Denmark as previously 331 described ${ }^{42,43}$. Each individual pig faecal sample was homogenized with a sterile wooden spatula 332 and then separated into two large aliquots. One of the aliquots was spiked with a freshly prepared 333 mock community consisting of 6 bacterial and 2 eukaryotic microorganisms, namely 334 Propionibacterium freudenreichii DSM 20271, Bacteroides fragilis NCTC 9343, Staphylococcus 335 aureus NCTC 8325, Fusobacterium nucleatum ATCC 25586, Escherichia coli ATCC 25922, 336 Salmonella Typhimurium str. ATCC 14028S, Cryptosporidium parvum IOWA II isolate, and Saccharomyces cerevisiae S288C. The spiked fecal samples were homogenized using a sterile wooden spatula, and small aliquots for each sample storage condition were prepared in Eppendorf tubes for both, the spiked and unspiked aliquots. DNA was isolated immediately from the aliquots for the initial time point (storage for Oh) using a modified QIAamp Fast DNA Stool Mini Kit (Qiagen) protocol with an initial bead beating step (MoBio garnet beads) ${ }^{44}$. The remaining aliquots were stored at different storage conditions comprising different temperature and time combinations (Figure 1A). Samples were stored at $22^{\circ} \mathrm{C}, 5^{\circ} \mathrm{C},-20^{\circ} \mathrm{C}$, and $-80^{\circ} \mathrm{C}$ for several hours (days) (16h (0.67 days), 40h (1.6 days), 64h (2.6 days), 88h (3.6 days)) and for the temperatures $-20^{\circ} \mathrm{C}$ and $-80^{\circ} \mathrm{C}$ also for months and up to one year $(4 \mathrm{~m}, 8 \mathrm{~m}, 12 \mathrm{~m})$. Aliquots stored for $40 \mathrm{~h}$ and $88 \mathrm{~h}$, as well as a subset of $64 \mathrm{~h}$, also underwent $2-4$ freeze-thaw cycles. All fecal samples underwent the same DNA isolation method (see above $)^{43,44}$ prior to library preparation.

Three different library preparation kits (the PCR-free NEXTflex and KAPA, as well as Nextera) and two sequencing platforms (HiSeq 4000 and NextSeq 500) were used in the study, similarly as previously described ${ }^{42,43}$. All samples were sequenced paired-end with a read length of 150 bp. Two to three technical replicates were performed for each treatment combination.

352 Together, a total of 184 different samples spread across 60 different replicate groups were 353 acquired, encompassing 21 specific combinations of storage conditions, spiking status, library 354 preparation kit used, as well as thawing cycles. The raw reads are deposited at the European 355 Nucleotide Archive (ENA) (Project acc.: PRJEB31650).

\section{Quality control and taxonomic classification}

357 Quality control of sequencing files was done following DTU Food's in-house pipeline, 358 FoodQCpipeline (https://bitbucket.org/RolfKaas/foodqcpipeline), in which BBMap's bbduk2 
(v38.71, https://jgi.doe.gov/data-and-tools/bbtools/) was used to trim reads with a length of at least $50 \mathrm{bp}$, Phred score of at least 20, and also remove a custom list of Illumina adapters. FastQC (v0.11.8) was also applied to the files before and after trimming to assess the quality of the reads $^{45}$.

363 Taxonomic classification was done with Kraken $2^{46}$, followed by taxa abundance re-estimation 364 using bracken ${ }^{47}$ at species level with read length of 150 and minimum taxa threshold of 1 . A 365 custom genomic index was used for the classification based on GTDB release $89^{48,49}$, which 366 includes 23,458 bacterial and 1,248 archaeal species. After merging individual reports, taxa 367 filtering was done based on CPM $>4$ in at least $15 \%$ of all samples. A total of 8,453 taxa were 368 included for downstream analysis.

\section{RUV-III-NB batch correction method}

370 The novel RUV-III-NB (https://github.com/limfuxing/ruvIIInb/) method extends the RUV-III method 371 that was previously developed for array-based gene expression data ${ }^{50}$. Instead of using linear 372 model, it uses a Negative Binomial-based generalized linear model (GLM) with log link function 373 to model the effect of wanted biological signals and unwanted variations on the sequencing count 374 at taxon-level. The method uses negative control taxa (i.e., taxa whose variations are assumed 375 solely due to unwanted variations) to estimate the latent unwanted factors, followed by the 376 utilization of replicate information to estimate the taxon-specific effect of these latent unwanted 377 factors on the variations of the sequencing count. Once these are estimated, RUV-III-NB uses 378 percentile-invariant adjusted count to output the sequencing count matrix that has been adjusted 379 or corrected for the unwanted variations. The percentile-invariant adjusted count was calculated 380 based on a modification version of the randomized quantile residual method ${ }^{51}$.

\section{Sensitivity and specificity of batch correction methods}

382 We compare the effectiveness of the following methods for removing unwanted variation: 383 ComBat $^{52}$, ComBat-Seq ${ }^{53}, \mathrm{RUVg}^{18}, \mathrm{RUVs}^{18}$, and RUV-III-NB (see RUV-III-NB subsection). 384 ComBat and ComBat-Seq both utilize known batch variable to remove batch effects from a 385 dataset, though the former allows normalized and/or log-transformed matrix as input, whereas the 386 latter requires raw integer count matrix as input ${ }^{52,53}$. RUVg, RUVs, and RUV-III-NB all utilize control features in estimating unwanted factors. Both RUVg and RUVs assume an underlying 388 Normal model and take normalized and/or log-transformed matrix as input, while RUV-III-NB only accepts integer count matrix; though both RUVs and RUV-III-NB require sample replicate information ${ }^{18}$. Out of all the methods, only ComBat-Seq and RUV-III-NB deal directly with the 
mean-variance relationship in the count data by using NB distribution. We tested two different logtransformation approaches for uncorrected data and prior to ComBat correction: normalization by library size / total sum scaling (TSS) followed by log2 transformation, as well as Aitchison's centered log-ratio (CLR) transformation ${ }^{54-56}$. Prior to RUVg and RUVs, CLR transformation was also performed. For RUV methods, we set $k$ - which represents the number of unwanted variations - to 7 as it is the highest possible number for the smallest set of control features. For RUV-III-NB, we also set the parameters lambda. $a=0.01$ and lambda. $b=5$.

In addition to the 8 spike-in taxa, we curated a set of empirical control taxa - which is defined as a set of microbial taxa that are present and empirically concordant in abundance in both groups that we would like to compare against - to be used as control features in RUV methods. To identify the empirical control taxa, the following procedure was used:

- Differential abundance analysis was done between spiked and unspiked samples using edge $\mathrm{R}^{57,58}$ for each unique replicate group of each pig. In average, only $<1 \%$ of the taxa were significantly differentially abundant in all the replicate groups.

- We then took the intersection of the least significant taxa from each replicate group within the same pig, specifically the bottom $86 \%$ of overall taxa based on FDR $<0.05$, as control taxa for each pig.

- From the control taxa of both pigs, we then took the intersection and identified a total of 471 overlapping taxa. This set is what we refer to as the empirical control taxa from this point on.

414 before and after correction to assess how well the different methods perform. RLE plots visualize

415 the presence of unwanted variations by calculating the deviations of from the median of each

416 feature, in this case microbial taxa, whereas PCA plots visualize clustering of certain batches 417 through unsupervised dimensionality reduction ${ }^{59,60}$. For RUV-III-NB, the log of percentile-invariant 418 adjusted count was used as the adjusted data matrix for the visualizations. To compare the RLE 419 plots between methods, we calculated a metric capturing the variance of medians of samples 420 from the same individual as well as the variance of the RLE's interquartile ranges (IQR):

421 - We subtracted each sample's median with the average median of all samples from the same pig source, then find the variance of all the medians. 
- After the addition of the variance of medians and the variance of all the IQRs from the RLE, we then performed log transformation to the total.

- We took the negation of the transformed value; hence, higher metric implies more effective removal of unwanted variations.

427 For PCA plots, we calculated the silhouette statistics using the 'cluster' package ${ }^{61}$ (version 2.1.0)

428 to assess two different comparisons:

- Preservation of individual source information, in which we calculated the score of separation between P1 and P2 samples and higher scores signify better preservation.

- Batch dispersion, in which we calculated the scores to see clustering of batch groups within each pig group, with lower scores signifying better removal.

We also performed an additional specificity assessment for the methods through differential abundance analysis using edge $\mathrm{R}^{57,58}$ on samples from the same source (P1) between groups of the main batch - in this case, between frozen and unfrozen storage conditions. Since ComBat only produces a transformed adjusted data matrix and not the estimated factors of unwanted variations, and therefore lacking the factors of unwanted variations necessary for covariates in differential abundance analysis, it is excluded from this analysis. Output from ComBat-Seq was directly used as input for the analysis. For RUV methods, untransformed integer counts were used as input along with factors of unwanted variations (W) as covariates in the linear model. Out of all the differentially abundant microbial taxa, we set the significance threshold to those with $p$ value $<0.05$ after FDR correction and with absolute log-foldchange of over 1 . We also performed $q$-value estimation of the results, which measures the proportion of true null $p$-values (pi0).

\section{Quantify relative contribution from unwanted factors}

We used RUV-III-NB to quantify unwanted factors in the data and analyze their relative contribution towards microbial taxa abundance. Hence, for our main analysis only spiked samples were used as the approach requires control features as input, which in our case included the 8 spike-in taxa and an additional set of 471 empirically constant taxa explained in the previous section. We set the same number of $k$ as stated in the previous section for consistency. We then used a negative binomial generalized linear model (glm.nb) and model the regression of each microbial taxa as the response variable and the progressive accumulation of factors of unwanted variations $W$ as the predictor. We then calculated the Pseudo- $R^{2}$ from each model using the PseudoR2 function from the 'DescTools' R package (version 0.99 .38$)^{62}$ with Veall-Zimmermann correction $^{63}$, as it is among the closest approximations to ordinary least square $\mathrm{R}^{2}$. Excluding the first factor, the contribution of each individual unwanted factor (W) towards each microbial taxon 
456 is represented by the difference in pseudo- $R^{2}$ between the cumulative $n^{\text {th }}$ and $n-1^{\text {th }}$ models (i.e. 457 contribution of W3 was calculated by subtracting the pseudo- $\mathrm{R}^{2}$ accounting for unwanted factors $458 \mathrm{~W} 1+\mathrm{W} 2+\mathrm{W} 3$ with the value accounting for unwanted factors $\mathrm{W} 1+\mathrm{W} 2)$. To see the effect of known 459 technical variations on microbial abundance, we then took the top 100 affected microbial taxa by 460 each of the individual factor of unwanted variations, ranked based on their pseudo- $R^{2}$ and 461 grouped based on taxonomic class. Since factor of unwanted variation W1 correlated strongly 462 with library size and geometric mean instead of any known technical variation, we only included 463 factors W2 - W7 in this step. From the top 100 affected microbial taxa in each factor of unwanted 464 variation, we calculated the proportion of their abundance in an average sample. For each of the 465 most dominantly affected microbial classes, we also performed Wilcoxon signed rank test 466 between storage conditions and Kruskal-Wallis test to compare the different freeze-thawing 467 cycles and library preparation kits on log2-transformed, TSS-normalized data. 


\section{References}

469 1. Morgan, X. C. \& Huttenhower, C. Chapter 12: Human Microbiome Analysis. PLoS Comput. Biol. 8, e1002808 (2012).

4712 2. Tilg, H. \& Moschen, A. R. Microbiota and diabetes: An evolving relationship. Gut 63, 1513-1521 (2014).

473 3. Proctor, L. M. et al. The Integrative Human Microbiome Project. Nature 569, 641-648 (2019).

475 4. Wang, J. et al. A metagenome-wide association study of gut microbiota in type 2 diabetes. Nature 490, 55-60 (2012).

5. Wong, A. C. \& Levy, M. New Approaches to Microbiome-Based Therapies. mSystems 4, (2019).

6. Lemon, K. P., Armitage, G. C., Relman, D. A. \& Fischbach, M. A. Microbiota-targeted

8. Nearing, J. T., Comeau, A. M. \& Langille, M. G. I. Identifying biases and their potential

9. McMurdie, P. J. \& Holmes, S. Waste not, want not: why rarefying microbiome data is inadmissible. PLoS Comput. Biol. 10, e1003531 (2014).

10. Byrd, D. A. et al. Comparison of Methods To Collect Fecal Samples for Microbiome Studies Using Whole-Genome Shotgun Metagenomic Sequencing. mSphere 5, (2020).

11. Ma, J. et al. Variations of Gut Microbiome Profile Under Different Storage Conditions and Preservation Periods: A Multi-Dimensional Evaluation. Front. Microbiol. 11, 972 (2020).

12. Pribyl, A. L. et al. Critical evaluation of faecal microbiome preservation using metagenomic analysis. ISME Commun. 1-10 (2021). doi:10.1038/s43705-021-00014-2

13. de Goffau, M. C. et al. Human placenta has no microbiome but can contain potential pathogens. Nature 572, 329-334 (2019).

14. Drago, L. et al. Microbiota network and mathematic microbe mutualism in colostrum and mature milk collected in two different geographic areas: Italy versus Burundi. ISME J. 11, 875-884 (2017).

15. Zuo, T. et al. Gut mucosal virome alterations in ulcerative colitis. Gut 68, 1169-1179 (2019).

16. Kennedy, K. M. et al. Fetal gut colonization: meconium does not have a detectable 
microbiota before birth. bioRxiv 2021.02.17.431710 (2021).

doi:10.1101/2021.02.17.431710

17. Leek, J. T., Johnson, W. E., Parker, H. S., Jaffe, A. E. \& Storey, J. D. The sva package for removing batch effects and other unwanted variation in high-throughput experiments. Bioinformatics 28, 882 (2012).

18. Risso, D., Ngai, J., Speed, T. P. \& Dudoit, S. Normalization of RNA-seq data using factor analysis of control genes or samples. Nat. Biotechnol. 32, 896-902 (2014).

19. Gibbons, S. M., Duvallet, C. \& Alm, E. J. Correcting for batch effects in case-control microbiome studies. PLoS Comput. Biol. 14, e1006102 (2018).

20. Rivera-Pinto, J. et al. Balances: a New Perspective for Microbiome Analysis. mSystems 3, e00053-18 (2018).

21. Gloor, G. B., Macklaim, J. M., Pawlowsky-Glahn, V. \& Egozcue, J. J. Microbiome

22. Townes, F. W., Hicks, S. C., Aryee, M. J. \& Irizarry, R. A. Feature selection and

23. Hafemeister, C. \& Satija, R. Normalization and variance stabilization of single-cell RNAseq data using regularized negative binomial regression. Genome Biol. 20, 296 (2019).

24. Wu, W. K. et al. Optimization of fecal sample processing for microbiome study — The journey from bathroom to bench. Journal of the Formosan Medical Association 118, 545555 (2019).

25. Shaw, A. G. et al. Latitude in sample handling and storage for infant faecal microbiota studies: The elephant in the room? Microbiome 4, 40 (2016).

26. Choo, J. M., Leong, L. E. X. \& Rogers, G. B. Sample storage conditions significantly influence faecal microbiome profiles. Sci. Rep. 5, 1-10 (2015).

27. Hickl, O. et al. Sample preservation and storage significantly impact taxonomic and functional profiles in metaproteomics studies of the human gut microbiome.

Microorganisms 7, (2019).

530 28. King, C. H. et al. Baseline human gut microbiota profile in healthy people and standard reporting template. PLoS One 14, e0206484 (2019).

532 29. Barelli, C. et al. The Gut Microbiota Communities of Wild Arboreal and Ground-Feeding Tropical Primates Are Affected Differently by Habitat Disturbance. mSystems 5, (2020).

30. Jiao, N. et al. Gut microbiome may contribute to insulin resistance and systemic inflammation in obese rodents: A meta-analysis. Physiol. Genomics 50, 244-254 (2018). 
31. Vujkovic-Cvijin, I. et al. Dysbiosis of the gut microbiota is associated with HIV disease progression and tryptophan catabolism. Sci. Transl. Med. 5, (2013).

32. Capuco, A. et al. Current Perspectives on Gut Microbiome Dysbiosis and Depression. Advances in Therapy 37, 1328-1346 (2020).

33. Kaakoush, N. O. et al. Microbial dysbiosis in pediatric patients with Crohn's disease. J. Clin. Microbiol. 50, 3258-3266 (2012).

34. Cheung, S. G. et al. Systematic review of gut microbiota and major depression. Frontiers in Psychiatry 10, 34 (2019).

35. Hevia, A. et al. Intestinal dysbiosis associated with systemic lupus erythematosus. MBio

36. Conesa, A. et al. A survey of best practices for RNA-seq data analysis. Genome Biology 17, (2016).

37. Zaheer, R. et al. Impact of sequencing depth on the characterization of the microbiome and resistome. Sci. Rep. 8, 5890 (2018).

38. Paulson, J. N., Bravo, H. C. \& Pop, M. Reply to: A fair comparison. Nat. Methods 11, 359-360 (2014).

39. McKnight, D. T. et al. Methods for normalizing microbiome data: An ecological perspective. Methods Ecol. Evol. 10, 389-400 (2019).

40. Dheda, K. et al. Validation of housekeeping genes for normalizing RNA expression in real-time PCR. Biotechniques 37, 112-119 (2004).

41. Eisenberg, E. \& Levanon, E. Y. Human housekeeping genes, revisited. Trends in Genetics 29, 569-574 (2013).

42. Poulsen, C., Pamp, S., Ekstrøm, C. \& Aarestrup, F. Library preparation and sequencing platform introduce bias in metagenomics characterisation of microbial communities. bioRxiv 592154 (2019). doi:10.1101/592154

43. Poulsen, C. S., Kaas, R. S., Aarestrup, F. M. \& Pamp, S. J. Standard Sample Storage Conditions Impact on Inferred Microbiome Composition and Antimicrobial Resistance Patterns. bioRxiv (2021). doi:10.1101/2021.05.24.445395

44. Knudsen, B. E. et al. Impact of Sample Type and DNA Isolation Procedure on Genomic Inference of Microbiome Composition. mSystems 1, 2021 (2016). Throughput Sequence Data. Available at: 
46. Wood, D. E., Lu, J. \& Langmead, B. Improved metagenomic analysis with Kraken 2. Genome Biol. 20, 257 (2019).

572 47. Lu, J., Breitwieser, F. P., Thielen, P. \& Salzberg, S. L. Bracken: Estimating species abundance in metagenomics data. PeerJ Comput. Sci. 2017, e104 (2017).

48. Parks, D. H. et al. A complete domain-to-species taxonomy for Bacteria and Archaea. Nat. Biotechnol. 38, 1079-1086 (2020).

49. Méric, G., Wick, R. R., Watts, S. C., Holt, K. E. \& Inouye, M. Correcting index databases improves metagenomic studies. bioRxiv 712166 (2019). doi:10.1101/712166

50. Molania, R., Gagnon-Bartsch, J. A., Dobrovic, A. \& Speed, T. P. A new normalization for Nanostring nCounter gene expression data. Nucleic Acids Res. 47, 6073-6083 (2019).

51. Dunn, P. K. \& Smyth, G. K. Randomized Quantile Residuals. J. Comput. Graph. Stat. 5, 236 (1996).

52. Johnson, W. E., Li, C. \& Rabinovic, A. Adjusting batch effects in microarray expression data using empirical Bayes methods. Biostatistics 8, 118-127 (2007).

53. Zhang, Y., Parmigiani, G. \& Johnson, W. E. ComBat-seq: batch effect adjustment for RNA-seq count data. NAR Genomics Bioinforma. 2, (2020).

54. Calle, M. L. Statistical Analysis of Metagenomics Data. Genomics Inform. 17, e6 (2019).

55. Aitchison, J. The Statistical Analysis of Compositional Data. (Chapman and Hall, 1986).

56. Quinn, T. P., Erb, I., Richardson, M. F. \& Crowley, T. M. Understanding sequencing data as compositions: An outlook and review. Bioinformatics 34, 2870-2878 (2018).

57. Robinson, M. D., McCarthy, D. J. \& Smyth, G. K. edgeR: a Bioconductor package for differential expression analysis of digital gene expression data. Bioinformatics 26, 139_ 140 (2010).

58. McCarthy, D. J., Chen, Y. \& Smyth, G. K. Differential expression analysis of multifactor RNA-Seq experiments with respect to biological variation. Nucleic Acids Res. (2012). doi:10.1093/nar/gks042

59. Gandolfo, L. C. \& Speed, T. P. RLE plots: Visualizing unwanted variation in high

600 61. Maechler, M. et al. Finding Groups in Data: Cluster Analysis Extended Rousseeuw et al. Cran (2019).

602 62. Signorell, A. DescTools: Tools for descriptive statistics. R package version 0.99.38. 
bioRxiv preprint doi: https://doi.org/10.1101/2021.05.21.445058; this version posted May 25, 2021. The copyright holder for this preprint (which

was not certified by peer review) is the author/funder, who has granted bioRxiv a license to display the preprint in perpetuity. It is made available under aCC-BY-ND 4.0 International license.

604 63. Veall, M. R. \& Zimmermann, K. F. Pseudo-R2 measures for some common limited

605 dependent variable models. J. Econ. Surv. 10, 241-259 (1996).

606

607 


\section{Authors' contributions}

609 AS, MI, and MF conceived the original project. AS and GM supervised the experiments. AS 610 constructed the tool highlighted in the study. SJP provided the microbiome dataset used in the 611 study. MF performed the experiments, analyzed the data, prepared the figures, and wrote the 612 bulk of the manuscript. GM, MI, AS, and SJP wrote and revised the manuscript. All authors read 613 and approved the manuscript.

\section{Acknowledgements}

615 Ml was supported by the Munz Chair of Cardiovascular Prediction and Prevention. MF was 616 supported by a Melbourne Research Scholarship from The University of Melbourne jointly funded 617 by the Baker Heart and Diabetes Institute. This work was supported by Health Data Research 618 UK, which is funded by the UK Medical Research Council, Engineering and Physical Sciences 619 Research Council, Economic and Social Research Council, Department of Health and Social 620 Care (England), Chief Scientist Office of the Scottish Government Health and Social Care

621 Directorates, Health and Social Care Research and Development Division (Welsh Government), 622 Public Health Agency (Northern Ireland), British Heart Foundation and Wellcome. This study was 623 also supported by the Victorian Government's Operational Infrastructure Support (OIS) program.

624 The funders had no role in study design, data collection and analysis, decision to publish, or 625 preparation of the manuscript. The views expressed in this manuscript are those of the author(s) 626 and not necessarily those of the NIHR or the Department of Health and Social Care.

\section{Data Availability}

628 The raw sequence data was deposited at the European Nucleotide Archive (ENA) under 629 accession number PRJEB31650. 


\section{Figures}
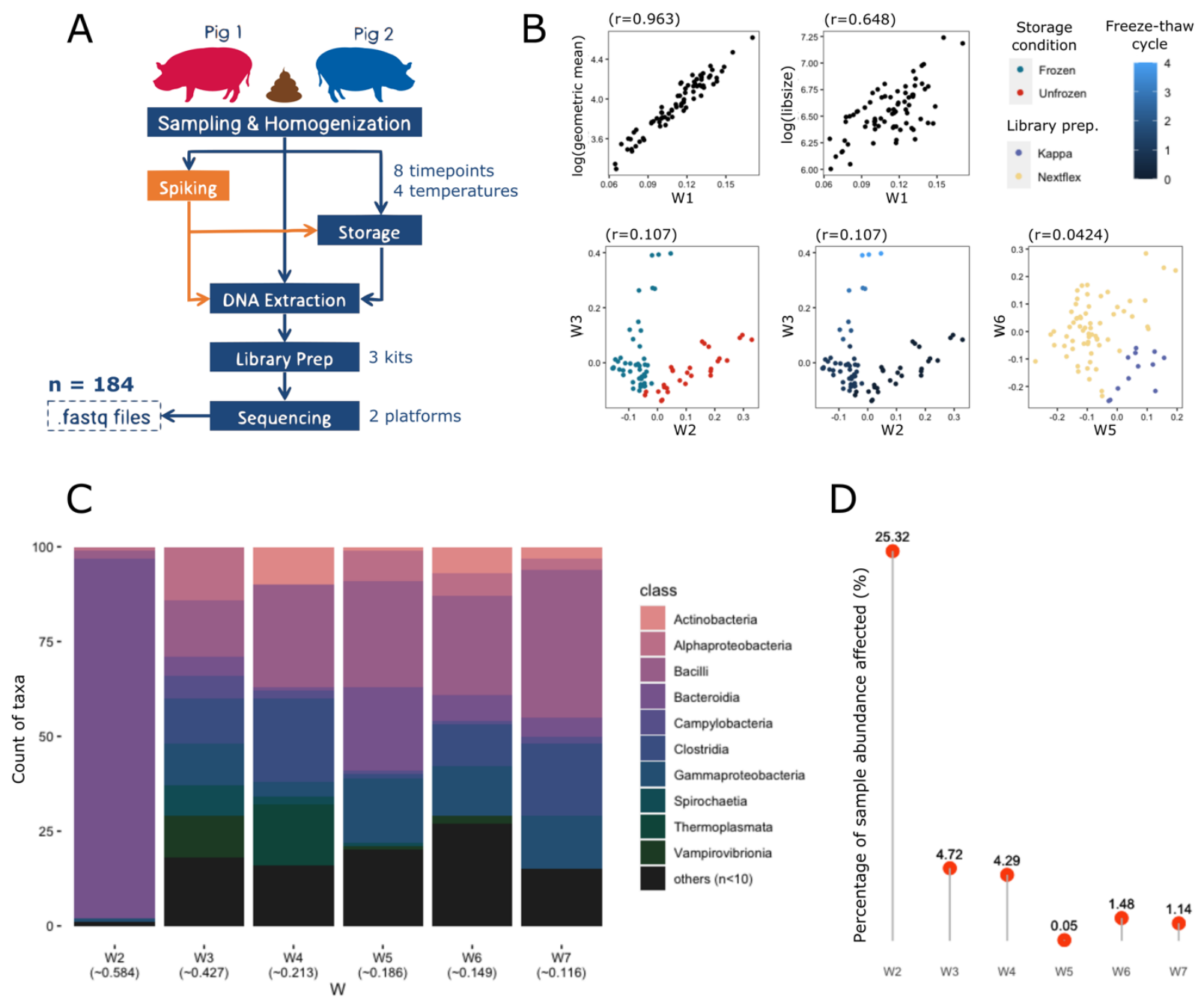

Figure 1: (A) Experimental workflow of the dataset, showing the sources of possible unwanted variations, some of which correlated with the calculated unwanted factors; (B) unwanted factor 1 (W1) correlated with log library size and log geometric mean, W2 correlated with storage conditions, W3 correlated with freezethaw cycles, and both W4 \& W5 correlated with library preparation kits. (C) The top 100 microbial taxa most affected by each $W$ colored by Class, ranked based on Veall-Zimmermann pseudo R-squared (mean listed under every W). Classes with less than two microbial taxa were merged as "others". (D) The proportion of a sample's total abundance affected by the top 100 microbial taxa for each W. 
A
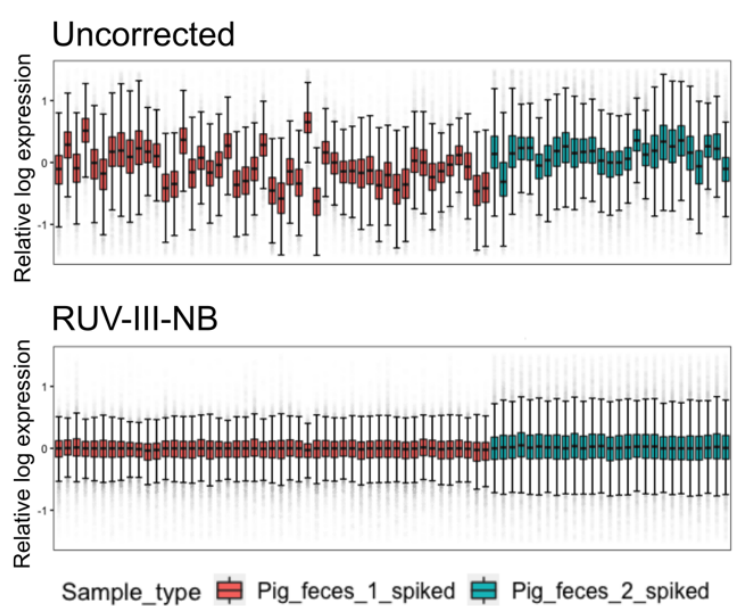

C

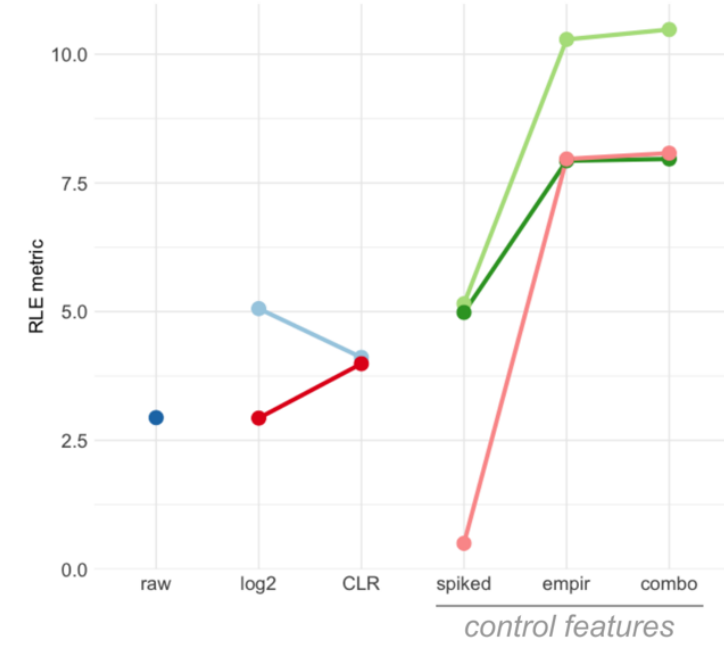

B
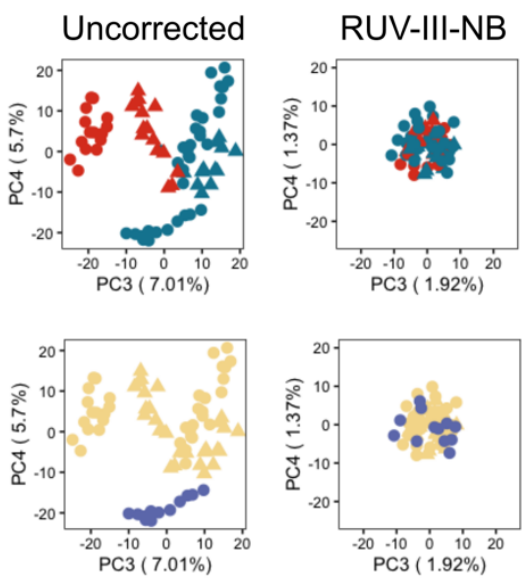

RUV-III-NB

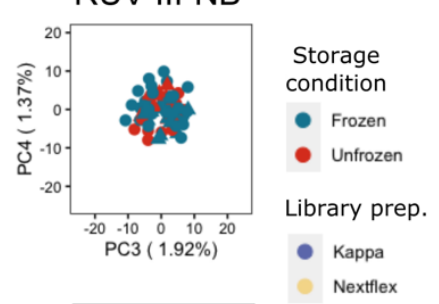

Sample type

- Pig_feces_1_spiked

- Pig_feces_2_spiked

Figure 2: (A) RLE plots of all spiked samples, before correction (top) and after RUV-III-NB correction with combination control taxa (bottom). In RLE plots, successful removal of unwanted variations is represented by the medians of sample from the same individual being as close to zero as possible and as linear to each other as possible, as well as similar interquartile ranges (IQR) between the samples visualized by the size of the boxplot. (B) PCA plots of the same set of samples, before (left) and after RUV-III-NB correction with combination control taxa (right). Clustering based on storage conditions and library preparation kits could be explained prior to correction by PC3 and PC4, respectively - which is no longer the case after correction. The lack of visible clustering suggests successful removal of unwanted variations. (C) Comparison of correction method performances based on relative log expression (RLE metric), where higher number indicates better removal of overall unwanted variations. With the exception of RUVs with solely spike-in taxa as control features, RUV methods in average performed better compared than ComBat-based methods. (D) Proportion of true null p-values (pi0) of uncorrected and corrected data using different correction methods after differential abundance analysis. Since storage conditions were found to be the main batch variable in this dataset, comparison was done between frozen and unfrozen samples of samples from the same source (pig 1). Since edgeR requires integer counts as input, ComBat was omitted from this comparison. RUV-III-NB resulted in the highest pi0 overall when using the combination set of control taxa, and still performed better than ComBat-seq when using solely empirical taxa as control features. 
A

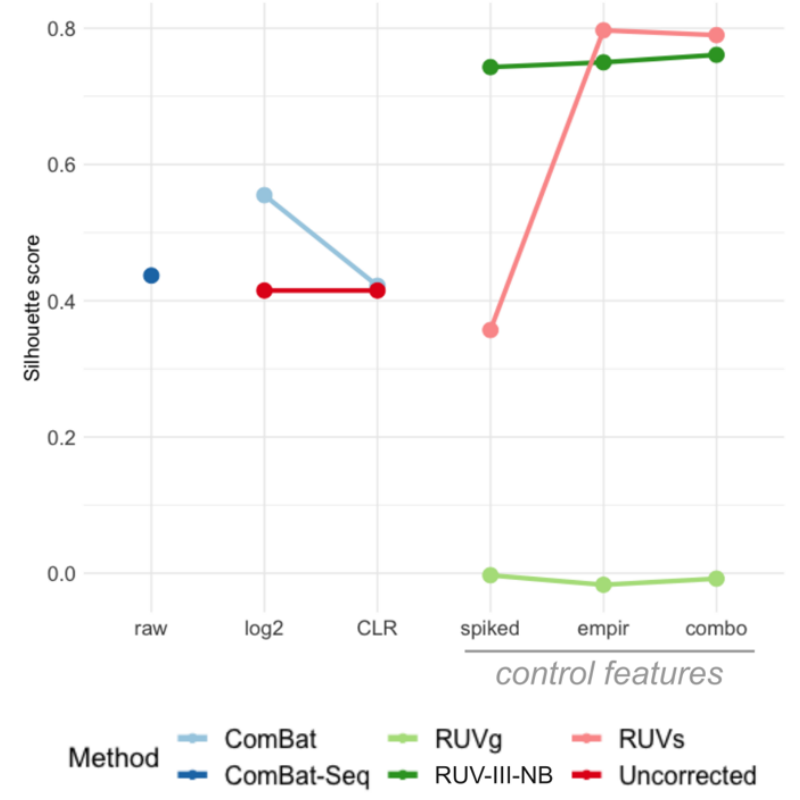

B

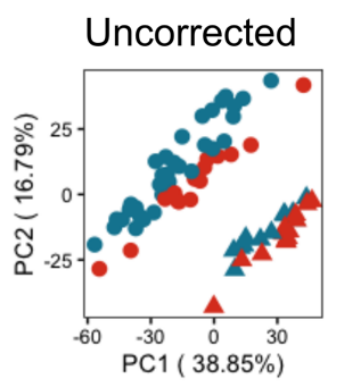

Storage condition

- Frozen

- Unfrozen

Sample type

- Pig_feces_1_spiked

A Pig_feces_2_spiked
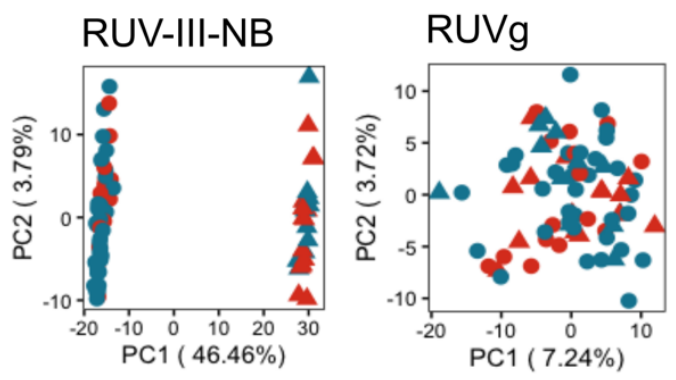

Figure 3: (A) Comparison of correction method performances in preserving individual biological information through silhouette scores, which are calculated based on the main principal components (PCs) of spiked samples and explain how well samples separate between defined groups (higher = better). RUVs using solely empirical control features had the highest silhouette score in separating pig 1 and pig 2 samples, yet correction using RUV-III-NB had the highest average silhouette score overall - indicating its consistency despite the different set of control features. RUVg performed really poorly overall, with silhouette scores using all the different control features placing significantly lower than even uncorrected data. (B) Based on the PCA plots of all spiked samples, separation of pig 1 and pig 2 samples can be explained by the main PCs in uncorrected data (top) and is even further defined after RUV-III-NB correction, clearly explained by PC1 (bottom-left). Separation of the two pig sources is lost after RUVg correction, as the two defined clusters are no longer visible. 


\section{Supplementary Materials}

\section{Supplementary Table}

673 Table S1: Results of differential abundance analysis of spiked pig 1 samples between storage conditions 674 (frozen vs unfrozen) using edgeR. Since all originated from the same individual and were spiked, little-to675 no taxa should be detected as differentially abundant. Out of 8,355 taxa, our analysis with ComBat-Seq 676 detected the lowest number of differentially abundant taxa (2) in the dataset, yet RUV-III-NB with combination control taxa resulted in the highest proportion of true null (pi0), indicating higher specificity.

\begin{tabular}{|c|c|c|c|c|c|}
\hline \multirow[b]{2}{*}{ Method } & \multirow[b]{2}{*}{ Variation } & \multicolumn{3}{|c|}{ Number of differentially abundant taxa } & \multirow{2}{*}{$\begin{array}{l}\text { Proportion } \\
\text { of true null }\end{array}$} \\
\hline & & $\begin{array}{c}\text { FDR }<0.05 \\
|\log 2(\mathrm{FC})|>1\end{array}$ & $\mathrm{p}<0.01$ & $p<0.05$ & \\
\hline Uncorrected & - & 273 & 3441 & 4418 & 0.317 \\
\hline ComBat-Seq & - & 2 & 60 & 425 & 0.905 \\
\hline$R U V g$ & spike-in control & 414 & 1585 & 2451 & 0.556 \\
\hline RUVg & empirical control & 67 & 446 & 1054 & 0.821 \\
\hline RUVg & combination control & 42 & 320 & 848 & 0.904 \\
\hline RUVs & spike-in control & 410 & 1602 & 2454 & 0.555 \\
\hline RUVs & empirical control & 35 & 263 & 733 & 0.861 \\
\hline RUVs & combination control & 31 & 215 & 637 & 0.955 \\
\hline RUV-III-NB & spike-in control & 363 & 1538 & 2520 & 0.622 \\
\hline RUV-III-NB & empirical control & 7 & 258 & 793 & 0.950 \\
\hline RUV-III-NB & combination control & 14 & 214 & 708 & 0.964 \\
\hline
\end{tabular}




\section{Supplementary Figures}
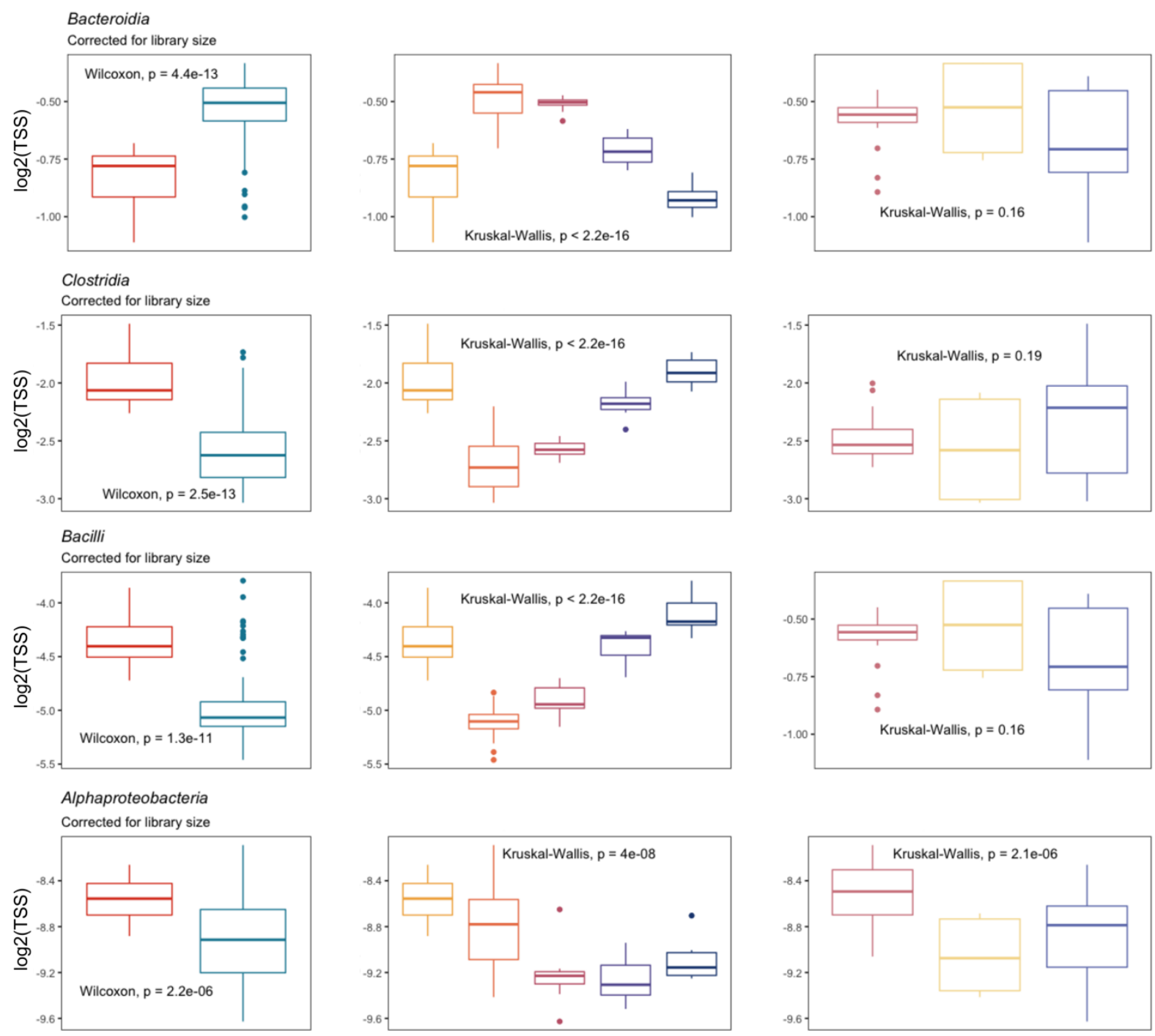

Storage 宊 Unfrozen 审 Frozen

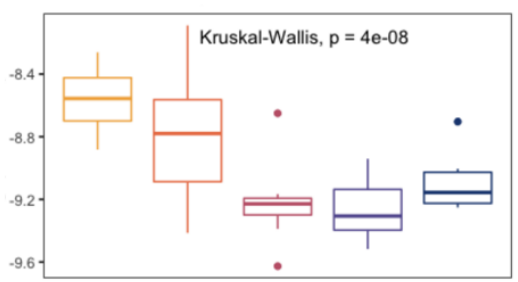

Freeze_thaw_cycles 宝 0 审 1 审 2 审 3 曻 4

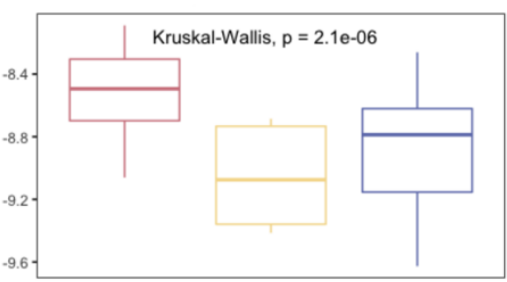

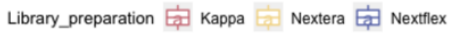

Figure S1: Differential abundance between variables of different batches (storage conditions, freeze-thaw cycles, and library preparation kits) in four of the most dominantly affected bacterial classes by unwanted differentially abundant between storage conditions and freeze-thaw cycles, with Alphaproteobacteria also being differentially abundant between library preparation kits. 
bioRxiv preprint doi: https://doi.org/10.1101/2021.05.21.445058; this version posted May 25, 2021. The copyright holder for this preprint (which was not certified by peer review) is the author/funder, who has granted bioRxiv a license to display the preprint in perpetuity. It is made available under aCC-BY-ND 4.0 International license.

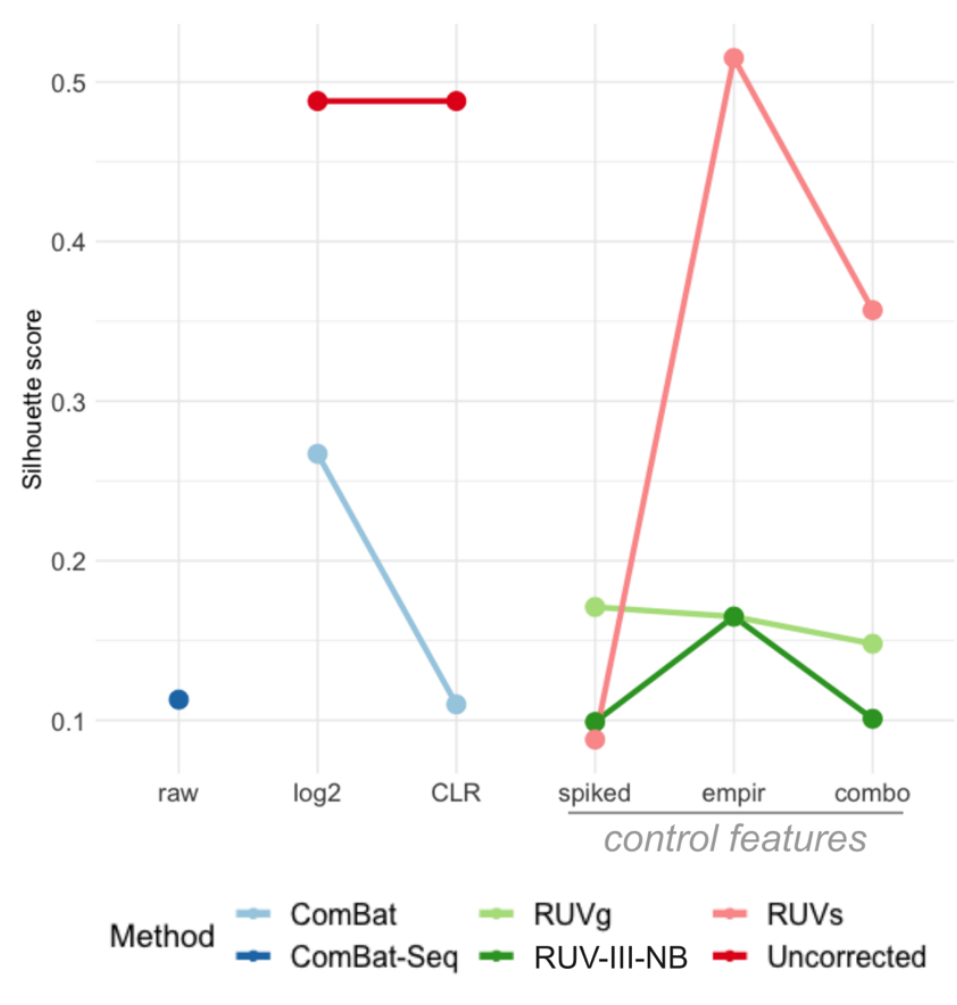

688 Figure S2: Comparison of correction method performances in removing unwanted variations from the main source (storage conditions). We calculated silhouette statistics based on the main principal components (PCs) of spiked samples, in which lower scores indicate poor clustering of batch variables, and therefore $(s s=0.51$ ), all approaches had lower silhouette scores compared to uncorrected data (uncorrected $s s=0.488$; ComBat $s s=0.11-0.26$; ComBat-Seq ss=0.11; RUVg ss=0.14-0.17; RUV-III-NB ss=0.09-0.16; RUVs ss=0.08-0.51). 



Figure S3: Results of differential abundance analysis of spiked pig 1 samples between storage conditions (frozen vs unfrozen), before (left) and after (right) correction using RUV-III-NB (with combination control features). Since the samples come from the same biological source, there should be little-to-no differences between the storage conditions. (A) volcano plot before correction shows over 200 significantly differentially abundant taxa (FDR $<0.05,|\log 2(\mathrm{FC})|>1)$, with only 14 differentially abundant taxa after RUV-III-NB correction. (B) The distribution of $p$-values before correction is anti-conservative, noted by the peak on the left due to all the differentially abundant taxa. A more uniform $p$-value distribution can be seen after RUVIII-NB correction, suggesting minimum number of differentially abundant taxa. 